\title{
Lapurdum
}

Euskal ikerketen aldizkaria | Revue d'études basques |

Revista de estudios vascos | Basque studies review

$20 \mid 2017$

Numéro $X X$

\section{Brèves notes sur une version bretonne peu connue du conte des deux frères (AT 303 The Twins or BloodBrothers)}

Herve Le Bihan

\section{(2) OpenEdition}

12 Journals

\section{Édition électronique}

URL : https://journals.openedition.org/lapurdum/3474

DOI : 10.4000/lapurdum.3474

ISSN : 1965-0655

Éditeur

IKER

\section{Édition imprimée}

Date de publication : 1 janvier 2017

Pagination : 45-56

ISBN : 978-2-95534-135-3

ISSN : 1273-3830

\section{Référence électronique}

Herve Le Bihan, «Brèves notes sur une version bretonne peu connue du conte des deux frères (AT 303 The Twins or BloodBrothers) », Lapurdum [En ligne], 20 | 2017, mis en ligne le 01 janvier 2021, consulté le 03 septembre 2021. URL : http://journals.openedition.org/lapurdum/3474 ; DOI : https://doi.org/ 10.4000/lapurdum.3474

Creative Commons - Attribution - Pas d'Utilisation Commerciale - Pas de Modification 4.0 International - CC BY-NC-ND 4.0 


\section{Brèves notes sur une version bretonne peu connue du conte des deux frères (AT 303 The Twins or Blood- Brothers)}

Herve LE BIHAN

Université Rennes 2, AOROC UMR 8546

Le corpus des contes bretons de langue bretonne a ceci de particulier que l'on ne considère plus que la masse de la collecte de François-Marie Luzel, constituée dans la deuxième moitié du XIX ${ }^{\text {ème }}$ siècle. Collecte qui a connu, très tôt, une version traduite en français. Elle est ainsi devenue la seule, ou presque, référence à laquelle se reporte la majorité des études lorsque le domaine bretonnant est sollicité. Les référencements de Paul Delarue et de ses continuateurs ne démentent en rien ce constat. Pourtant ce corpus est loin d'être exhaustif et d'être le seul ayant une valeur intrinsèque. Depuis longtemps d'autres chercheurs ont pu montrer que d'autres corpus de contes bretons possèdent cette même valeur ${ }^{1}$.

Le but de ces quelques lignes est d'attirer l'attention sur une version peu connue, remarquable à notre point de vue, d'un conte en langue bretonne.

Le conte en question est tiré du recueil Kountadennou «Contes» publié par Christophe Jézégou à Châteaulin en 1909. Recueil dont aucune traduction n’a encore été publiée.

Christophe Jézégou (1864-1953) était prêtre originaire du Léon (grosso-modo le nord du département du Finistère) et exerça son ministère à Chateaulin pendant une période assez longue, période qu'il mit à profit pour collecter ses contes. Merlik «Merlin», dont il sera

1. Comme, par exemple, les études produites par Claude Sterckx. 
question ici, est une conte appartenant au terroir de Chateaulin. D'autres versions, mais bien moins remarquables ont été collectées dans le même secteur. Yeun ar Gow, notaire à Pleyben, avait lui aussi collecté ce conte et l'avait publié en 1939, sous le titre de Marc'heger ar Gergoad «Le chevalier de Kergoat».

Le conte Merlik, comme beaucoup de contes, rassemble plusieurs types de récits. On peut le découper en deux grandes parties. La première fait référence à l'homme sauvage (AT 502 The Wild Man) en mettant en scène le personnage de Merlin, thème récurrent depuis les récits du Moyen Âge dans tout le monde brittonique. On sait que le personnage de Merlin, concentrant sur lui les vertus d'anciens dieux liés à la nature, est commun aux traditions galloise, bretonne et cornique, et qu'il a vu le jour dans le sud de l'Écosse, qui était alors de langue brittonique.

La seconde fait référence nettement au conte des deux frères (AT 303 The Twins or Blood-Brothers) $)^{2}$. Ce type de conte est très répandu mais avec des évolutions plus ou moins grandes. C'est à ce type que l'on rattache toute une série de contes intitulés «Le pêcheur et ses fils» (souvent au nombre de trois). Le corpus basque en donne au moins une version donnée par la collecte Webster (2005, p. 135-143).

Ce conte des deux frères est réputé être le conte le plus vieux du monde. La version la plus ancienne a été collationnée sur un papyrus égyptien. Cependant les versions modernes sont assez éloignées du modèle égyptien (Schuler, 1999).

Il y a aussi interférences entre les deux types de contes au sein du récit de Merlik. Par exemple les fils du pêcheur sont les filles du marquis.

Ce qui nous semble remarquable dans ce conte est l'ambigüité constante portant sur l'identité sexuelle des personnages dans la première partie : le personnage du cavalier blanc est une femme (costumée en homme) dont tombe amoureuse la reine, mais aussi le roi. Et une fois la reine éliminée, le roi prendra pour femme le cavalier blanc (redevenu femme dans son aspect).

Une autre ambigüité apparaît également dans la deuxième partie, nettement dominée par la gemellité des deux héros : il est assez clair que les deux demoiselles accueillent de la même manière le premier frère puis le second, et ce, en croyant (ou en feignant de croire ?) avoir affaire au même personnage. Et le conte se termine par la distributivité des deux héros avec les deux demoiselles sans qu'il n'y ait de souci sur la reconnaissance de l'un ou de l'autre.

Enfin ce qui est sans doute le plus surprenant est de voir qu'un tel conte a pu être publié ainsi par un écclésiastique. On doit se poser la question de la réception d'un tel conte (dans sa version imprimée, écrite) auprès d'un public qui était sous la coupe règlée de l'Église à cette époque.

2. Voir aussi le conte $N^{\circ} 60$ donné par la collecte des frères Grimm. 


\section{Texte de la traduction}

Merlig $^{3}$

Autrefois la haute noblesse devait toujours avoir un fils au palais du Roi.

Et voici qu'un marquis n'avait pas eu de fils. Mais, cependant, il avait trois filles. du Roi

Et il eu l'idée un jour d'habiller une d'entre elles en homme et de l'envoyer au palais

Il en parla à sa fille aînée.

- O ! Bien sûr, dit-elle, j’irai avec joie jusqu'au palais du Roi.

On lui sella un des plus beaux chevaux. Elle dit au-revoir à ses parents : elle prit la route.

Elle avait revêtu l'habit d'un homme et elle avait l'air d'un cavalier.

Mais son père avait peur que sa fille ne soit pas assez courageuse. Il prit de mauvais habits et mit un chapeau sur sa tête qui cachait son visage, et il prit à travers par les champs pour se poster sur le chemin que sa fille devait prendre.

Alors qu'elle passait il fit un saut à la tête du cheval et il prit la bride en disant avec une voix menaçante : « La bourse ou la vie !».

La pauvre fille en devint blanche comme un morceau de papier, elle jeta sa bourse sur le sol et détala vers la maison.

Le marquis l'avait précédée.

Lorsqu'il la vit dans la cour, il fit mine d'être navré :

- Tiens, dit-il, tu es de retour?

- O ! Oui, dit-elle, en ce qui me concerne je n'irai pas au palais du Roi : il y a des voleurs sur le chemin. J'ai dû leur laisser ma bourse sinon ils auraient pris ma vie.

- Bah! dit le marquis.

Et il eu l'idée d'envoyer la deuxième de ses filles.

Il lui fit le même tour. Et elle prit aussi peur comme sa sœur aînée.

Puis il demanda à la dernière si elle aurait peur des voleurs.

- O ! Non, assurément, dit-elle. Si vous le désirez j'irai jusque la demeure du Roi pour tenir l'honneur de notre nom. Cependant j'aimerais ne pas y aller avant huit jours.

- Très bien! dit le marquis, vas-y d'ici à huit jours.

Et durant ces huit jours là elle alla, sans rien dire à son père, à la ville la plus proche acheter un pistolet.

Lorsque le jour indiqué arriva, elle dit au-revoir et se mit en route.

Le marquis prit encore des vêtements pouilleux et il coupa court devant elle comme il avait fait devant ses sœurs. Il la vit arriver. Le cheval dansait sous elle. Et elle, sans souci aucun,

3. La version originale porte la forme Merlik, forme qui se normalise aujourd'hui en Merlig. 
tenait les rênes des deux mains.

Et voilà, tout d'un coup, le vieux marquis à la tête du cheval en criant : la bourse ou la vie!

Et elle, avec rapidité, d'attraper son pistolet de la sangle de la selle et de tirer.

Par chance le marquis ne fut pas atteint. Il découvrit sa tête: Très bien ma fille, dit-il, tu as le courage d'un homme: je vois que mon sang pur coule dans tes veines. Au palais du Roi tu feras honneur à notre nom. Pars donc à l'aventure de Dieu !

Ils s'embrassèrent une dernière fois. Le père s'en vint chez lui et elle continua son chemin.

Arrivée au palais du Roi elle lui donna une lettre qu'elle avait obtenue de son père. Le Roi qui connaissait le vieux marquis lui donna le meilleur des accueils.

- Quel est votre nom ? lui dit-il.

- Mon nom, dit-elle, est le cavalier blanc. Les gens du palais lui firent un très bon accueil : elle était si mignonne qu'elle attirait l'amitié de tous.

La Reine elle-même demanda à ce qu'on lui attribue le cavalier blanc en tant que page.

Elle avait un amour anormal pour elle. Elle était à sa suite dans la chambre comme dans n'importe quel endroit. Mais le cavalier blanc, que voulez-vous, lui était indifférent.

Puis elle en devint jalouse. Elle lui reprocha tant de choses mauvaises que le Roi en vint à l'emprisonner.

Une nuit, alors qu'elle ne pouvait dormir à cause du grand chagrin qu'elle avait en pensant au mal que la Reine lui avait fait, elle vit une dame venir à elle, lumineuse :

- Cavalier blanc, dit-elle, n'ayez ni peur ni crainte. La Reine vous a fait jeter ici par jalousie. Mais je viendrai, contre elle, pour vous apporter aide. Demain vous serez condamnée à capturer Merlig.

- Merlig ? dit la jeune femme, qu'est-ce que Merlig ? ${ }^{4}$

- Un homme avec deux cornes sur la tête et qui vit dans le grand bois. Il fait beaucoup de dégâts et de chamboulements par là-bas. Il a tué, jusqu'ici, quiconque essayait de le capturer.

Mais lorsqu'il vous sera dit que vous devez y venir à bout, voilà ce que vous ferez.

Tout d'abord vous demanderez une cage en fer, une grande, et plusieurs sortes de bonbons pour mettre dans cette cage de fer. Ensuite vous demanderez un chaudron qui contienne l'équivalent d'une barrique d'eau. Et ensuite un bœuf, un veau, une barrique de vin et un sac rempli de moineaux.

Grâce à toutes ces choses vous capturerez Merlig et voici comment.

Lorsque vous serez arrivée dans le bois, vous mettrez la cage de fer à côté d'une fontaine qui s'y trouve, au pied d'un chêne couvert de lierre.

Ensuite mettez de l'eau de la fontaine dans le chaudron et déchargez la barrique de vin dans la fontaine. Puis vous placerez le veau et le bœuf dans le chaudron et vous ferez du feu

4. Le texte original porte petra eo Merlik ? tournure qui ne s'utilise qu'avec des êtres inanimés ou des êtres animés mais non considérés comme humains (animaux, enfants en bas âge, etc). 
dessous pour les cuire. Ouvrez la porte de la cage de fer et mettez y en évidence les bonbons à l'autre bout.

Lorsque vous tout arrangé ainsi, allez vous cacher dans le chêne, au milieu du lierre.

Merlig sentira l'odeur de la viande cuite et viendra. Mais comme il est rusé il en aura crainte. Avant de s'emparer de quoi que ce soit il regardera partout. Il lancera des pierres, même, contre l'arbre pour voir s'il n'y a pas du monde de caché parmi le lierre. Mais à chaque fois vous laisserez s'échapper quelques moineaux.

Les choses se passèrent comme elle le lui avait dit.

Elle fut condamnée à capturer Merlig.

Elle demanda ce qu'avait indiqué la dame.

Le Roi qui pensait l'envoyer à la mort comme tous ceux qui avait cherché à capturer Merlig satisfit ses demandes. De plus il lui proposa un sifflet et lui dit en se moquant:

- Tiens : lorsque tu auras capturé Merlig tu n'auras qu'à siffler. Nous entendrons du palais et nous irons te récupérer immédiatement.

Arrivé dans le bois, le cavalier blanc répartit les choses comme cela lui avait été ordonné : faire la soupe, mettre le vin dans la fontaine, ouvrir la cage de fer, ranger les bonbons, et placer le sac de moineaux dans l'arbre.

Bientôt elle vit un homme immense recouvert de crin qui lui faisait un manteau assez semblable à celui d'une chèvre. Deux énormes cornes garnissaient sa tête et il regardait ici et là autour de lui avec méfiance.

Arrivé devant la fontaine il se baissa pour boire de l'eau. Lorsqu'il eu bu il mis sa main sur le ventre en disant :

- L'eau est bonne aujourd'hui !

Mais il n'était absolument pas rassuré. Il regardait avec inquiétude autour de lui :

- Là, dit-il, il y a sûrement des gens dans l'arbre, cachés parmi le lierre.

Et il attrapa des pierres pour les jeter sur l'arbre.

Le cavalier blanc ouvrit le sac et laissa échapper un vingtaine de moineaux.

- Tiens donc, dit Merlig, des oiseaux : il n'y a pas d'êtres humains qui soient des oiseaux.

Et il s'approcha pour manger la soupe.

Ce ne fut pas long.

Ensuite il alla voir ce qu'il y avait dans la cage de fer.

- Des bonbons! Dit-il. A ! je parie qu'il y a des humains par ici et ils cherchent à me faire un mauvais tour!

Et il examina les buissons alentours, et il jeta à nouveau des pierres contre l'arbre.

Le cavalier blanc laissa encore de nombreux moineaux s'échapper.

- O ! Des oiseaux, dit Merlig, il n'y a personne ici.

Et il pénétra dans la cage de fer.

Mais il n'y rentra pas complètement : il recula.

- C'est sûr, dit-il, il doit y avoir quelqu'un qui cherche à me trahir.

Et il examina encore le bois et il jeta des pierres contre l'arbre, une nouvelle fois.

Le cavalier blanc laissa à nouveau des moineaux s'échapper. 
- O ! Là au moins, il n'y a personne, là il n'y a que des oiseaux.

Et il pénétra dans la cage.

Aussitôt, en un bond vif, le cavalier blanc était au sol face à la cage et il en verrouilla la porte.

Merlig, dans une grande colère, remua et fit du bruit de manière épouvantable. Mais hélas! Il était pris et bien pris.

Le cavalier blanc attrapa alors son sifflet et s'en servit trois fois.

Le Roi appela et accompagné de tous les gens de son palais il se précipita pour voir ce qu'il en était.

Ils firent un nombre incommensurable de remerciements reconnaissants au cavalier blanc : ils en étaient fiers.

La cage de fer fut mise sur une charrette : tout autour on avait placé vingt gens d'arme pour surveiller Merlig. Le cavalier blanc alla au palais, transporté dans une voiture entre le Roi et la Reine.

Alors qu'il était en route, Merlig grinçait des dents et frappait les barreaux de sa cage en criant : «Ah! Si j'avais su que cette vieille fille ${ }^{5}$ aurait réussi à me capturer, je l'aurais tuée, je l'aurais dévorée. »

Et les gens d'arme se disaient entre eux :

- Ecoute ce que dit Merlig!

- Oui, oui, disait Merlig, c'est une jeune femme qui m'a capturé ; et j'en suis sûr, c'est une jeune femme vierge.

- Eh bien, dirent les gens d'arme, il faudra en parler au Roi.

Arrivés au Palais, les gens d'arme contèrent au Roi ce qu'ils avaient entendu.

Le Roi en fut navré.

Le lendemain il y avait un grand repas en l'honneur du cavalier blanc.

Après le repas on alla faire un tour aux jardins. Le Roi se fit accompagner de son médecin et il l'entretint sur les propos de Merlig.

Juste à ce moment là le cavalier blanc passait en compagnie d'un officier.

Le médecin jeta un regard sur lui et dit :

Eh bien, si j'avais réfléchi plus tôt j'aurais dit comme Merlig : il est certain que le cavalier est une femme.

Alors, le Roi appela le cavalier blanc pour qu'il l'accompagne jusqu'à sa chambre ${ }^{6}$. Il lui conta ce qu'il avait entendu.

Le cavalier blanc se mit à pleurer, pensant qu'il allait être expulsé.

- O ! Ne pleurez pas, dit le Roi. Je vois à présent, que la Reine m’a conté beaucoup de

5. Le texte original porte an tamm koz plac'h iaouank-se, mot à mot « ce bout de vieille jeune femme » où koz antéposé a un rôle dépréciatif.

6. Il s'agit de la chambre du Roi. 
mensonges à votre propos. J'ai de la haine pour elle : je ne lui pardonnerai jamais.

Et le jour suivant, il fit chauffer le four et y fit jeter la Reine pour la brûler.

Cependant le Roi demanda aux nobles du palais ce qu'il fallait faire de Merlig. Tous dirent qu'il fallait le maintenir dans sa cage de fer et mettre la cage de fer au milieu de la cour du palais.

- La clé, dirent-ils, sera attachée par une chaîne d'or au coup du cavalier blanc et celui qui jamais ouvrira la cage sera mis à mort.

Par suite le Roi eut envie de se marier, à nouveau. Il jeta son dévolu sur la cavalier blanc.

Les noces eurent lieu. Et l'année suivante il y eut un enfant, fort comme son père et tout mignon comme sa mère.

Lorsqu'il commença à marcher on le laissait dans la cour devant la demeure ; on lui avait donné des boules d'or pour jouer. Les boules roulaient jusqu'à la cage de Merlig. Merlig les attrapaient et les lançaient au petit garçon.

Un jour, cependant, ils les garda toutes.

- Ramène moi mes boules, disait le petit garçon.

- Non, tant que tu n'auras pas ouvert la porte de ma cage, disait Merlig.

- Je ne sais, disais le petit garçon, où en est la clé.

- Au cou de ta mère, attachée par une chaîne d'or. Ta mère est endormie dans sa chambre, va la chercher.

Le garçon alla jusqu’à la chambre de sa mère, attrapa la clé et libéra Merlig.

Merlig jeta ses boules à l'enfant et il s'échappa promptement du palais en lâchant un tel cri qu'il en résonna aux alentours.

La Reine se réveilla, troublée.

- O ! Dit-elle, Merlig s'est échappé ! Mais qui a pu lui ouvrir?

Son petit garçon qui était en sa présence lui dit :

- C'est moi ma mère, pour récupérer mes boules.

- O ! Pauvre petit malheureux! Dit la Reine, maintenant tu vas être mis à mort.

Le Roi vint jusqu'à la chambre de la Reine. Tous deux pleuraient en pensant à ce qu'avait fait leur enfant.

Les juges, cependant, trouvait l'enfant trop jeune pour être exécuté. Ils le condamnèrent, seulement, à l'exil, toute sa vie durant.

Dans le port, devant le palais, il y avait un navire étranger.

Le Roi et la Reine demandèrent au capitaine de prendre leur enfant et de l'amener dans les pays lointains.

- Restez en sa compagnie, dirent-ils. Nous vous payerons. Mais ne lui dites jamais quel est le nom de ses parents ni celui de son pays.

- Oui, dit le capitaine. C'est avec bonne volonté que je le prendrai comme mon enfant. Je n'ai plus de famille et il me sera facile de m'attacher à lui.

Environ deux années plus tard, le navire se trouvait dans un port espagnol. L'enfant, assez grand pour alors, jouait sur le pont du navire. 
Il arriva alors que la Reine d'Espagne regarde par la fenêtre.

- Vois, dit-elle, en appelant le Roi, où est aller notre fils pour jouer.

Et eux de convoquer la jeune fille chargée de leur fils et de la réprimander.

- O ! Dit-elle, cessez, votre enfant est ici. Et elle leur apporta leur enfant.

Ils allèrent accompagnés de leur fils pour voir s'ils retrouveraient l'enfant du navire semblable à leur enfant.

- Allons voir, dirent-ils.

Et eux, lorsqu'ils arrivèrent, de demander au capitaine à qui était cet enfant.

- Hélas! Dit le marin, je ne peux absolument pas vous dire qui il est : mais j'en ai pris la charge.

- Eh bien, dit le Roi, vous êtes âgé à présent. Vous ne pourrez l'élever, c'est presque sûr. Donnez le nous. Nous l'élèverons en compagnie du notre et nous vous dédommagerons.

- O ! Répondit le capitaine, je vous remercie. J'ai assez pour vivre de ce que les parents de l'enfant m'ont donné. Je vous suis cependant reconnaissant de ce que vous me proposez, et c'est de bon cour que je vous laisserai l'enfant.

L'enfant quitta donc le navire pour rejoindre le palais du Roi d'Espagne. Il était fier d'avoir un enfant de son âge pour jouer avec lui.

Alors qu'ils grandissaient, les deux enfants devenaient de plus en plus semblables. Le Roi et la Reine ne pouvaient plus les reconnaître l'un de l'autre.

Cependant, lorsqu'il devint jeune homme, l'orphelin dit à son ami :

- On m'a dit que je ne suis pas d'ici. J'ai envie de connaître mes parents. Moi, maintenant, je vais aller de pays en pays pour les rechercher.

- O, dit le fils du Roi d'Espagne, je vais t'accompagner.

- Non ; tu ne peux pas. Tu briserais le cœur de ton père et celui de ta mère. Tu resteras ici. Mais, tous les jours, va au jardin cueillir une feuille du laurier. Prend une épingle pour percer cette feuille. Le jour où en sortira du sang cela dira que je suis en danger. Viens rapidement, alors, pour me chercher.

Le Roi et la Reine d'Espagne, lorsqu'ils entendirent que l'ami de leur fils s'en allait, furent très inquiets.

- Comment, lui dirent-ils, tu n'es pas heureux ici?

- O ! Si, très heureux, trop heureux, dit-il. Je vous remercie de tout cœur. Mais j’ai envie de voir si je pourrai retrouver mes parents.

On lui donna un cheval plus léger que le vent.

Il s'en alla droit devant lui...

Après avoir parcouru et parcouru encore, il se retrouva face à l'océan. Mais il était inquiet. Il ne savait pas comment traverser. l'aide.

Il lui vint le souvenir, je ne sais comment, de Merlig, et voilà qu'il appelle Merlig à

Merlig entendit l'appel et arriva aussi vite que le vent :

- Que te manque-t-il ? Dit-il.

- J'ai envie de traverser la mer, dit le jeune homme...

- Ah! Ce n'est que ça. 
Et Merlig transporta sur son dos le cheval et le jeune homme et les amena l'autre côté de la mer, sur le sec.

- Au-revoir! Dit-il.

Et en partant il lâcha un cri tel que la mer et la terre en résonnèrent.

Le jeune homme alla de nouveau devant lui.

Il atteignit un château. Les portes en étaient closes. Et c'était déjà la nuit. Il n'osait pas aller plus loin et il demanda à ce qu'on lui ouvrit.

Une jeune demoiselle des plus jolies vint lui ouvrir :

- Soyez le bienvenu, Monseigneur! Dit-elle. Cela fait tellement longtemps que nous n'avons vu personne! O ! Ici tout le monde en sera heureux !

On prépara le meilleur des dîners en son honneur.

Après dîner, en l'accompagnant à sa chambre, la demoiselle dit :

- N'ayez pas peur si vous entendez la cloche sonner à minuit. Mon frère, dit-elle, doit aller, chaque nuit, combattre une vieille sorcière. Chaque nuit il la tue, et chaque matin elle revient à la vie.

- Ah! Vraiment? Dit le jeune homme. J'aimerais savoir qu'elle est cette sorcière.

- O ! Il ne faut pas que vous alliez voir, dit la demoiselle. Plus d'un y ont été voir et ont été tués.

Cependant, lorsqu'il entendit le cloche dans la nuit, il se leva de son lit. Il prit son pistolet et il alla se cacher derrière un buisson d'épines.

Le frère de la demoiselle tua la sorcière et la mit en pièces.

- Si elle revient désormais à la vie, je dois voir comment.

Sous peu il vit venir une autre vieille sorcière. Elle avait une fiole à la main.

- Oh ! Ma pauvre petite sœur, disait-elle, tu as encore été tuée !

Et elle rassemblait les morceaux de sa sour. Et alors qu'elle les rassemblait, elle les rattachait les uns aux autres avec le produit contenu dans sa fiole.

Le jeune homme leva son pistolet et tira sur la vieille sorcière. Il la détruisit et lui pris sa fiole. Il resta un moment à épier pour voir si une autre sorcière viendrait rendre la vie aux deux autres. Mais personne ne vint.

Le matin il dit à la demoiselle :

- Votre frère n'aura sans doute plus besoin de veiller comme auparavant. J'ai tué la sorcière qui redonnait vie à la sorcière tuée par votre frère.

- Oh ! Ce n'est pas complètement sûr qu'elle reste morte, dit la demoiselle. Ce soir on verra si la cloche ne sonne pas. Restez encore dormir au château. Vous verrez si elle ne revient plus.

La nuit suivante, il avait beau attendre, aucune cloche ne sonna.

Le jeune homme dit à la demoiselle :

- Vous voyez que ce que je vous ai dit est vrai.

- Oh! Quel service, dit-elle, nous avez-vous rendu là. Restez ici, désormais avec nous.

- Hélas! Je ne peux rester. Je suis à la recherche de mes parents.

Et il leur conta son histoire. 
- Eh bien, dit la demoiselle, puisque vous devez partir, vous partirez. Mais nous avons des cousines à quelque distance d'ici. Vous irez les voir pour leur demander le nom de vos parents. Nous leur écrirons et vous serez reçu du mieux par elles.

Le jeune homme repris à nouveau son chemin.

Et voici qu'il arriva devant un manoir magnifique. C'était le manoir des cousines de la demoiselle du château. Il fut très bien reçu. Il y eut un grand repas en son honneur et lorsque la nuit vint on l'amena dormir dans une chambre magnifique.

Le lendemain matin, il était en train de regarder par la fenêtre les belles choses qu'il y avait autour du manoir lorsque vint l'une des demoiselles lui demander comment c'était passée sa nuit.

- Oh! Très bien, mademoiselle. Je vous remercie. Et comme vous avez de belles choses autour de ce manoir! Je dois aller voir le pré qui est là-bas, les fleurs qui y sont sont si belles.

- Oh! Au nom de Dieu! N'y allez pas, dit la demoiselle. Quiconque y va est tué.

Mais le jeune homme était si attiré par les fleurs qu'il voyait, qu'il sauta par la fenêtre pour atteindre le pré dès que la demoiselle s'était retirée.

Il était en train de cueillir des fleurs lorsqu'un vieil homme surgit devant lui. Il avait une faucille à la main, et avant que le jeune homme ne puisse se protéger, il fut décapité.

Ce matin là, comme d'habitude, son ami, le fils du Roi d'Espagne, vint cueillir une feuille de laurier. Lorsqu'il la transperça il vit du sang en venir.

Et il alla trouver son père et sa mère et très tristement il leur dit :

- Il est arrivé malheur à mon ami : je dois aller à sa recherche.

Et il prit la route le plus rapidement possible.

Après quelques jours de voyages il arriva au château où son ami avait été reçu tout d'abord. Il demanda à ce qu'on lui ouvre. La demoiselle vint lui ouvrir :

- Oh ! Dit-elle, aussitôt qu'elle le vit, que je suis heureuse de vous voir. Je ne puis vous remercier assez de nous avoir délivrés de la vieille sorcière.

Le fils du Roi d'Espagne pensa :

- Mon ami est passé par là.

Mais il ne corrigea pas les dires de la demoiselle.

- Oui, dit-il, vous êtes donc heureuse de me voir ?...

On l'amena dans la maison. Le frère lui fit aussi bon accueil.

Il le prenait aussi pour son ami.

Le lendemain il leur dit qu'il devait partir.

- Déjà, dirent-ils. Vous n'avez encore retrouvé vos parents?

- Eh non, répondit-il. Mais plus tard je reviendrai encore. Au revoir!

Et il se mit en route.

Le lendemain il se retrouva devant un manoir.

Et il frappa à la porte.

La demoiselle qui était venue lui ouvrir sautait de joie :

- Magnifique, dit-elle, vous êtes revenu ! J'avais eu tellement de chagrin l'autre jour, votre chambre vide. Sûrement que vous resterez avec nous désormais ?...

- Mon ami est venu ici aussi, pensa le fils du Roi d'Espagne. 
Lorsque le soir vint on lui montra la chambre où il irait dormir. Et il alla à la fenêtre.

- Oh, dit-il, que les fleurs sont belles!

- Ainsi donc, dit la demoiselle, toujours attiré par les fleurs. Lautre fois je vous disais la même chose. Il ne faut pas aller voir. Quiconque descend jusqu'aux fleurs est tué.

- Mon ami est là, pensa le fils du Roi.

Mais il ne dit rien.

Lorsque la nuit fut venue, il avait hâte d'aller jusqu'au champ de fleurs. Sur le bord non labouré du champ il découvrit son ami décapité.

Il se mit, je ne sais à cause de quoi, à chercher dans ses poches et il trouva la fiole du produit de la vieille sorcière.

Il frictionna ce produit sur la tête et le corps de son ami. Il eut le plaisir de voir la tête et le corps se souder et de voir son ami revenu à nouveau à la vie.

Aussitôt debout il dit au fils du Roi d'Espagne:

- Partons vite d'ici.

Et ils rentrèrent dans le manoir par la fenêtre.

Ils s'étaient à peine faufilés à l'intérieur, que le vieux sorcier était près de la fenêtre avec sa faucille. Mais cette fois là il avait été trop tardif.

Le jour d'après la demoiselle vint le matin pour saluer le jeune homme.

Elle fut surprise lorsqu'elle vit deux au lieu d'un et que les deux étaient semblables l'un à l'autre.

Le fils du Roi d'Espagne lui raconta leur l'histoire.

Au repas, par la suite, le jeune orphelin demanda la main de la demoiselle du château.

Le fils du Roi d'Espagne alla à l'autre manoir où il avait d'abord été reçu pour demander la sienne.

Les noces, vous pouvez le croire, furent faites immédiatement.

Et jamais ils ne furent plus heureux.

Cherchez un bon ami, aimez-vous tous les jours.

Et si vous êtes bon votre peine sera alors plus légère.

C'est dans les mauvaises périodes que les amis

Sont reconnus.

\section{Bibliographie}

Aarne, Antti \& Thompson, Stith. 1964. The Types of the Folktale. Helsinki: Suomalainen Tiedeakatemia.

ar Gow, Yeun, Marc'heger ar Gergoad, $3^{e}$ édition, hor Yezh, 2013. [première édition en 1939]

Belmont, Nicole (édit.), Emmanuel Cosquin - Contes, Arles, 2003.

Canadé Sautman, Francesca, Conchado, Diana \& Di Scippio, Giuseppe Carlo, Telling Tales Medieval Narratives and the Folk Tradition, New York, 1998.

Cerquand, Jean-François, Légendes E récits populaires du Pays Basque, Bedous, 1996. 
Colum, Padraic, Campbell, Joseph \& Scharl, Josef (édit.), Jacob and Wilhelm Grimm / Complete Fairy Tales, London \& New York, 2007.

Delarue, Paul, Le conte populaire français, Paris, 1985.

Jackson, Kenneth, The International Popular Tale and Early Welsh Tradition, Cardiff, 1961.

Jezegou, Kristof, Kountadennou livet ha renket, Librairie Aimé Corcuff à Chateaulin, [1909].

Martin, Jean-Pierre, L'autre conte des deux frères, in «Les avatars d'un conte», Communications, No39, Paris, 1984, p. 190-193.

Propp, Vladimir, Morphologie du conte, Paris, 1970.

Rimasson-Fertin, Natacha (édit.), Contes pour les enfants et la maison collectés par les Frères Grimm, tome 1, José Corti, Paris, 2009.

Sébillot, Paul, Contes de la Haute-Bretagne, Paris, 1892.

Schuler, François (Edit.), Conte des deux frères suivi de Le mari trompé, traduit des hieroglyphes et postfacé, $2^{e}$ édition, Jose Corti, 1999.

Simonsen, Michèle, Le conte populaire français, Paris, 1986.

Soriano, Marc, Les contes de Perrault - Culture savante et traditions populaires, Paris, 1977.

Sterckx, Claude, Mythologie du monde celte, Marabout, 2009.

Webster, Wentworth, Légendes basques, recueillies principalement dans la province du Labourd, Mayenne, 2005.

Zaïka, Natalia M., Approche textologique et comparative du conte traditionnel basque dans les versions bilingues de 1873 à 1942 (W. Webster, J.-F. Cerquand, J. Barbier, R. M. de Azkue), Iker, No 31, Bilbao, 2104.

Zipes, Jack (édit.), Brothers Grimm / The Complete Fairy Tales, London, 2007. 